\title{
Aspekte der Lehrplangestaltung unter Berücksichtigung der regionalen Geographie
}

\section{Faktoren der Lehrplangestaltung}

Gesellschaftliche Faktoren spiegeln die Zeitströmungen wider. Lehrpläne der 70er Jahre sollten zum Aufbau einer rationalen, wissenschaftsgeprägten Welt beitragen. In der Geographie galt es, allgemeingeographische Strukturen zu erkennen, aus Fallbeispielen Modelle abzuleiten, die globale Gültigkeit haben sollten. In den 80er Jahren wurde dem Thema Umwelt große Bedeutung eingeräumt, die Gefährdung thematisiert. In den $90 \mathrm{er} J a h$ ren werden längst überwunden geglaubte Themen wie der Nationalismus wieder bedeutsam, neue regionale Kräfte wie der politisch wirkende Islam müssen berücksichtigt werden.

$\mathrm{Zu}$ den gesellschaftlichen Faktoren gehört die Frage, ob ein Lehrplan stärker Wissen oder mehr Qualifikationen zu vermitteln habe. Stärker konservativ geprägte Regierungen betonen das Allgemeinwissen, für die Geographie mag hier das Schlagwort «Topographie» stehen; wer sich als progressiv bezeichnet, betont Qualifikationen wie die Arbeit mit Karten oder die Fähigkeit, sich so zu verhalten, daß Räume unter größtmöglicher Schonung der Natur die Ansprüche möglichst vieler gesellschaftlicher Gruppen befriedigen.

Erziehungswissenschaftliche Faktoren sind oft eng mit den gesellschaftlichen verknüpft (vgl. etwa KLAFKI 1991, HASSE 1994). So wird gefordert, statt Fachwissen fächerübergreifende Qualifikationen zu vermitteln. Themen wie Umwelterziehung, Friedenserziehung, "Eine Welt» oder informationstechnische Bildung sollen verstärkt behandelt werden. Dies führt in einigen Ländern dazu, daß Geographie in ein neues Fach integriert wird, das etwa "Welt- und Umweltkunde» heißt und die bisherigen Fächer Geographie, Geschichte und Sozialkunde zusammenfaßt. Eine wichtige didaktische Forderung (vgl. dazu HENNINGS 1993) ist im Schweizer Rahmenlehrplan bereits verwirklicht: der Lehrplan darf nicht zu viel vorgeben, muß dem einzelnen Lehrer einen möglichst groBen Entscheidungsrahmen lassen.

Geowissenschaftliche Faktoren spielen seit den 70er Jahren, als die Sozialgeographie in der Schule der Entwicklung in der Wissenschaft oft vorauseilte, eine geringere Rolle. Rein geowissenschaftliche Themen wie die Plattentektonik sind selten geworden, andererseits hat die regionale Geographie in der Schule eine größere Bedeutung als an der Universität.

Administrative Faktoren wirken auf den Lehrplan oft stärker ein, als es Außenstehenden bewußt wird. Das können konkrete Vorgaben sein, wie die Integration der Geographie in neue Fächer oder die verstärkte Berücksichtigung politisch aktueller Fragestellungen. Das können scheinbar nebenbei gegebene Hinweise sein, etwa, daß ein umfassendes topographisches Wissen dem Ansehen des Faches in der Öffentlichkeit nützt.

Persönliche Faktoren spielen ebenfalls eine Rolle, schließlich wird ein Lehrplan von Menschen verfaßt. Böse Zungen behaupten: «Ein Lehrplan wird von Lehrern gemacht, die anderen Lehrern zeigen wollen, wie schlau sie selbst sind.» Solcher Ehrgeiz spiegelt sich oft in wissenschaftsüberfrachteten Formulierungen, die weniger auf die Vermittelbarkeit in der Schule abzielen als auf das Erstaunen des Lehrers.

Es ist für den einzelnen Lehrer wichtig zu wissen, welche Faktoren einen Lehrplan beeinflussen. Es macht ihn gegenüber manchen Zielsetzungen gelassener, bestärkt ihn, geänderte Bedingungen der Gesellschaft, der Wissenschaft, seiner Klasse zu berücksichtigen.

\section{Aufgaben des Lehrplans}

\subsection{Wissen}

Ein Lehrplan legt fest, was ein Schüler wissen sollte, wobei es sowohl um den Umfang wie die Tiefe des Wissens geht. Am Anfang des Lehrplans stehen die Ziele, die «eigentlich» erreicht werden sollen, etwa "Handlungsfähigkeit in einer komplexen Welt», "Bereitschaft zum Schutz der Umwelt» oder "Raumverhaltenskompetenz». Sie sind teilweise überfachlich, notwendigerweise abstrakt. Die pädagogische Forderung geht dahin, die konkreten Inhalte aus den abstrakten Zielen abzuleiten. Vorbemerkungen im Lehrplan bemühen sich oft, eine Deduktion der konkreten Inhalte aus den abstrakten Zielen nachzuweisen. In der Praxis sind die Inhalte oft normative Setzungen. Denn man kann zwar noch begründen, warum man die Beziehungen der Schweiz zur EU behandeln sollte, auch noch Kenntnisse der Raumplanung, aber schon die Behandlung der Plattentektonik läßt sich nur schwer aus abstrakten Zielen ableiten. Manche Lehrplä-

Dieter Böhn, Prof. Dr., Lehrstuhl für Didaktik der Geographie, Universität Würzburg, Wittelsbacherplatz 1, D-97074 Würzburg 
ne legen auch die Begriffe fest, die erarbeitet werden sollen (z. B. City, Dritte Welt, Flächennutzungskonkurrenz, Migration, Tropen). Allerdings geschieht das recht selten, denn über Definitionen und Umfang eines Begriffskatalogs herrscht noch größere Uneinigkeit als über den Umfang topographischen Wissens.

\subsection{Fertigkeiten}

Ein wichtiges Ziel des Geographieunterrichts ist es, Fertigkeiten einzuüben, mit denen der Schüler sich jetzt und in Zukunft selbst Wissen erschließen, Fakten auf ihre Richtigkeit überprüfen und auf ihre Relevanz für sich oder die Gemeinschaft bewerten kann. Dazu gehören im Geographieunterricht instrumentale Fertigkeiten wie die Fähigkeit, mit Karten handelnd umzugehen (vom Stadtplan bis zur Wetterkarte) oder Daten, Bilder und Texte auswerten zu können. Hierzu gehört auch die Fähigkeit, sich über ein Land, ein Gebiet selbst einen Überblick zu verschaffen.

\subsection{Werthaltungen}

Alle Lehrpläne weisen darauf hin, daß ihr Ziel nicht Wissen an sich ist, sondern der Aufbau von Werthaltungen (Einstellungen, Qualifikationen), nach denen der Schüler dann im späteren Leben handelt. Der Schweizer Rahmenlehrplan nennt etwa den "verantwortungsbewußten Umgang mit dem Lebensraum» oder die Grundhaltung, «Begegnungen mit anderen Menschen, Kulturen und Landschaften als Bereicherung zu erfahren». Die Schwierigkeit in der Praxis besteht darin, daß man zwar durch das Wissen letztlich Werthaltungen erzielen will, aber ebenso letztlich nur Wissen abprüfen kann. Denn wie weit affektive Lernziele vom Schüler internalisiert werden, können wir in der kurzen Schulzeit nur vermuten. Wir haben zwar Chancen, weil wir die Menschen in dem Lebensabschnitt in der Schule haben, in denen sie sich Werthaltungen aufbauen. Doch stehen die in der Schule vermittelten Wertsysteme im Wettbewerb mit anderen, die etwa über die Werbung, durch das Elternhaus oder durch Jugendliche oft unterschwellig, aber sehr wirkungsvoll angeboten werden. Man kann zudem in der Schulzeit nur ein Fundament legen, die Menschen entwickeln sich (glücklicherweise) weiter, müssen zu neuen Aufgaben neue Antworten finden. Wir können nur hoffen, daß die - etwa im Geographieunterricht - vermittelten Werte ihre Entscheidungen beeinflussen.

\section{Möglichkeiten des Lehrplans}

Aus dem Lehrplan erwachsen dem Lehrer zwei große pädagogische Aufgaben, die gleich wichtig sind: Wissen zu vermitteln und Werte aufzubauen. Ideal ist es, wenn man durch Wissen erziehen kann. Während Ziele und Inhalte des Lehrplans oft recht verbindlich vorgegeben sind, hat der Lehrer bei der methodischen Umsetzung große Freiheiten. Diese sollte er nutzen. Es geht darum, den Schüler möglichst eigenständig lernen zu lassen (Lernen durch Handeln). So kann man etwa bei einem geographischen Planspiel (z. B. Anlage einer Fußgängerzone, Ausweisung eines Naturschutzgebietes, Standort einer Chemiefabrik) oder durch Werkstattarbeit (s. Beitrag Reinfried in diesem Heft, außerdem REINFRIED 1994) neben der Fähigkeit einer Bewertung von Raumstrukturen, Machtfaktoren und Umwelteinflüssen auch die Fähigkeit anbahnen, raumwirksame Entscheidungen so zu treffen, daß man die Argumente des anderen ernst nimmt, und neben rationalen Gesichtspunkten (wie Güterabwägung, Interessenausgleich und langfristige Entwicklung) auch emotionale zu akzeptieren (wie Heimatverbundenheit, persönliche Vorlieben und Abneigungen).

Planspiele, Projekte und Werkstattarbeit sind zwar arbeits- und zeitaufwendig (und daher nur begrenzt einsetzbar), aber die so erarbeiteten Themen bleiben dem Schüler im Gedächtnis. Hierbei lernt er, wie man auch später Fragestellungen angehen kann, wie man kreative Lösungen findet. Viel weniger zeitaufwendig und daher oft einzusetzen sind handlungsorientierte Tätigkeiten wie Arbeiten mit Karten, Texten, Statistiken, Diagrammen - alles Qualifikationen für das Leben -, aber auch Experimente.

Wenn immer möglich, sollte die Arbeit vor Ort erfolgen. Der Schüler lernt die ihm vertraute Umgebung unter einem neuen Blickwinkel kennen. Er lernt, wie man sich, etwa durch Kartierungen und Befragungen, selbst Unterlagen für die Beurteilung eines Sachverhalts erstellen kann. Er erkennt, daß verschiedene Möglichkeiten durchaus in sich schlüssig sind und daß es oft sehr schwer ist, eine Entscheidung zu fällen. Eine Arbeit vor Ort, welche die Stellungnahmen möglichst vieler Betroffener und Interessierter einbezieht, wird zu dieser wichtigen Erkenntnis gelangen: es gibt kein Richtig oder Falsch «an sich» (wie es von den meisten Befragten behauptet wird), es gibt nur ein "Besser» oder "weniger Gut» unter bestimmten Gesichtspunkten. Eine solche Erkenntnis, am konkreten aktuellen Beispiel vor Ort erarbeitet, trägt entscheidend zum Verständnis demokratischer Entscheidungen bei. Vor allem wird klar, wie schwer es ist, die «richtige» Entscheidung zu fällen. Und das wiederum macht toleranter.

\section{Geographische Inhalte des Lehrplans}

Wo Geographie als eigenständiges Fach erhalten bleibt und nicht unter scheinbar "übergeordneten" Ideen wie "Schlüsselqualifikationen» in ein schwer zu definierendes Fach integriert wird (z. B. KÖCK 1993, SCHMIDT-wULFFEN 1994), muß geklärt werden, unter welcher Leitidee geographisches Wissen vermittelt wird. Bis zu Beginn der 70er Jahre war dies klar: Geographie war identisch 
mit Länderkunde. Seitdem streiten sich die Geographiedidaktiker. In Deutschland haben diejenigen die Oberhand, die Geographie fast ausschließlich unter allgemeingeographischen Themen vermitteln wollen (vgl. z. B. KROSS 1992, BIRKENHAUER 1995). Anhänger der faktenorientierten, idiographischen und eine gewisse Vollständigkeit anstrebenden alten Länderkunde gibt es nicht mehr, doch wird von vielen eine stärkere Berücksichtigung regionaler Strukturen verlangt (vgl. z. B. BÖHN 1988, STORKEBAUM 1990, NEWIG 1995).

In der Praxis der Lehrpläne werden beide Auffassungen teilweise integriert, allgemeingeographische Strukturen werden an regionalen Beispielen aufgezeigt. Dabei werden z. B. in Bayern (LEHRPLÄNE BAYERN) in den Klassen 5 und 6 die allgemeingeographischen Strukturen gelegt, Beispiele dafür sind Stadt und Umland, Landwirtschaft, Industrie und Fremdenverkehr. In der 7. Jahrgangsstufe wird Europa behandelt, doch werden aus den einzelnen Staaten lediglich wenige Themen ausgewählt (z. B. bei Italien der Tourismus und der Nord-Süd-Gegensatz, bei Frankreich Zentrum und Peripherie, bei Nordeuropa Holz, Fisch und Erdöl). Der Schüler erfährt kaum etwas über die Länder als komplexe Gebilde. Nur wenige Staaten werden als Individuen erarbeitet. Es sind vor allem die USA (in der 9. Klasse), früher oftmals im Vergleich mit der Sowjetunion. Daneben wird in einigen Lehrplänen Indien, in anderen China und/oder Japan behandelt (z. B. 8. Klasse).

\section{Zur Notwendigkeit regionalgeographischer Ansätze}

Es gibt zahlreiche einleuchtende und schlüssige Gründe, warum die Allgemeine Geographie inhaltlicher Schwerpunkt des Erdkundeunterrichts sein sollte. Es ist andererseits unbestreitbar, daß regionalgeographische Kenntnisse unerläßlich sind. Die Welt besteht aus Staaten, und trotz zahlreicher internationaler Strukturen und Prozesse, die im Zuge der weltweiten Verflechtungen zunehmen, können politische und soziale Entwicklungen auf der Welt nicht verstanden werden, wenn wir nicht die regionalgeographische Komplexität erkennen.

Es ist klar, daß nicht alle Staaten der Erde, ja nicht einmal alle Europas im Erdkundeunterricht behandelt werden können. Welche Staaten ausgewählt werden, hängt von vielen Faktoren ab. Da die Staaten wechseln, über die sich ein Schüler, noch mehr später der Erwachsene, Informationen erarbeiten, diese bewerten und sich ein Bild machen sollte, können niemals alle Staaten im Unterricht im nötigen Umfang behandelt werden. Es ist notwendig, dem Schüler Schemata zu vermitteln, mit deren Hilfe er sich selbst Kenntnisse verschaffen kann (z. B. länderkundliches Schema, auch Hettnersches Schema genannt, oder Schema nach BÖHN 1988). Solche Schemata müssen im Unterricht geübt werden, damit man später mit ihnen selbständig arbeiten kann. Hier sollen lediglich einige Grundregeln angeführt werden.

\section{Grundregeln}

\section{zur Erarbeitung regionaler Strukturen und Prozesse}

1. Jede Untersuchung geht von einer bestimmten Frage aus, es gibt keine Untersuchung "an sich».

2. Für die Erlangung eines ersten Überblicks ist ein Schema hilfreich (z. B. länderkundliches Schema/Hettnersches Schema, Schema Böhn).

3. Informationen sind aus Karten, Statistiken, der Literatur z. T. leicht zugänglich.

4. Zahlreiche Informationen erlauben nur einen Überblick über den gegenwärtigen Zustand (genauer: den $\mathrm{Zu}$ stand zur Zeit der Datenerfassung). Sie ermöglichen weder einen Trend in die Zukunft noch in der Vergangenheit begonnene Entwicklungen zu erkennen.

5. Statistische Informationen geben oft nur einen ersten Einblick, vor allem Durchschnittswerte sind zu hinterfragen.

6. Viele Informationen aus Statistiken und Karten ermöglichen Tatbestände zu erkennen. Einen wirklichen Einblick erhält man aber nur, wenn auch die (raum)wirksamen Kräfte erfaßt werden.

7. (Raum)wirksame Kräfte sind unter anderem: Machtstrukturen (z. B. Art des Staatsaufbaus, der Regierung), grundlegende Gesellschaftsstrukturen (z. B. Agrargesellschaft, Industriegesellschaft, Informationsgesellschaft), Wirtschaftsstrukturen (z. B. Marktwirtschaft, Ausmaß staatlicher Eingriffe, regionaler Umfang der Wirtschaftsverflechtungen), allgemeine oder regionale Wertsysteme. 8. Erste Aufgabe ist die Erfassung der Daten, Strukturen und Prozesse. In einer weiteren Stufe müssen sie bewertet werden. Dabei gibt es grundsätzlich zwei Möglichkeiten:

- Bewertung aus der regionalen Situation selbst heraus

(z. B. auch Berücksichtigung der dort herrschenden Wertsysteme)

- Bewertung von übergeordneten Wertsystemen aus (z. B. unsere Auffassung unveräußerlicher Menschenrechte)

Grundsätzlich sollte zumindest in einer ersten Stufe von den im Untersuchungsgebiet herrschenden Wertvorstellungen ausgegangen werden, weil sonst ein Verständnis der vorhandenen Strukturen und der ablaufenden Prozesse nicht möglich ist.

\section{Beispiele der Realisierung des regionalen Ansatzes}

\subsection{Die geographische Erfassung der Heimat (des Nahraums)}

Heimat wird hier ohne ideologischen Ballast als der Raum definiert, in dem der Mensch lebt, in dem er objektiv seine wichtigsten wirtschaftlichen und sozialen Beziehungen unterhält, in dem er sich subjektiv «daheim» fühlt.

7.1.l Die Notwendigkeit, sich mit der Heimat zu befassen Heimat ist der Raum, in dem man lebt und handelt. Ihn zu kennen ist 
- eine Erfüllung der grundlegenden Neugier des Menschen für seine Umwelt,

- eine wirtschaftliche Notwendigkeit, weil die Situation in der Heimat Beruf und soziale Stellung beeinflußt,

- eine politische Forderung, denn in der Demokratie ist man gefordert, an der Gestaltung der Heimat mitzuwirken.

\subsubsection{Schwierigkeiten, sich mit der Heimat zu befassen}

Gerade weil die Heimat der Raum ist, den man am besten kennt, ergeben sich oftmals beträchtliche Schwierigkeiten. Sie führen (leider) dazu, daß zahlreiche Lehrer eine genauere Untersuchung des heimatlichen Raumes vermeiden oder ohne Schwerpunktsetzung durchführen. Solche Schwierigkeiten sind unter anderem

- die erkennbar hohe Komplexität der Raumstruktur. Während bei entfernten Räumen durch eine verminderte Wahrnehmung geographische Strukturen scheinbar einfach zu erfassen sind, fallen im Nahraum selbst Schülern die vielfachen Verflechtungen auf (z. B. zwischen der Anlage von Fußgängerzonen und den politischen Mehrheitsverhältnissen im Stadtrat, den Einwirkungsmöglichkeiten der Geschäftsleute, Parkmöglichkeiten im Nahbereich sowie der wirtschaftlichen Konkurrenz in Nachbarorten).

- der hohe Vorbereitungsaufwand. Fast nie steht ein Erdkundebuch zur Verfügung, Materialien müssen mühsam beschafft werden, sie veralten erkennbar schneller als solche aus fernen Räumen. Soll der Nahraum nach den gebräuchlichen geographiedidaktischen Methoden erarbeitet werden, sind Exkursionen bzw. Unterrichtsgänge erforderlich.

- Furcht vor politischen Schwierigkeiten. Geographisches Arbeiten vor Ort enthüllt oft, daß Raumstrukturen aus und zu sehr persönlichen Motiven verändert werden. Das aber wird von den «Mächtigen» oft nicht gern gesehen. Untersuchungen über Baugebiete, die Umwelt oder über Verkehrsführungen können zu solchen politisch bedingten Schwierigkeiten führen.

Es ist wichtig, dem Schüler auch die Schwierigkeiten der Raumgestaltung zu zeigen. Er muß erkennen, wie kompliziert Entscheidungen sind, wie viele Faktoren abzuwägen sind, wie es Mut erfordert, sich zu engagieren. Der Schüler muß diese Schwierigkeiten bereits im «Schonraum Schule» erfahren. Das bewahrt ihn später vor Frustration und politischer Abstinenz. Dies wiederum ist unerläßlich für ein demokratisches Staatswesen.

7.1.3 Methodische Verfahren bei der Erfassung der Heimat Gerade die Arbeit im Nahraum ermöglicht Erlernen und Einüben vielfältiger geographischer Arbeitsweisen. Beispiele dafür sind:

- Arbeiten mit Karten und Plänen

- eigene Kartierungen (z. B. funktionale Nutzung von Gebäuden, Bewertung von Gebäuden für bestimmte Funktionen wie den Fremdenverkehr, Nutzung von Flurstücken)

- Befragungen
- fotografische Erfassung geographischer Strukturen

- Erfassung naturgeographischer Daten (Klima, Relief, Boden)

- Untersuchungen zur Umweltqualität (Messungen von Wasser, Luft, Lärm)

\subsection{Die geographische Erfassung von Fernräumen}

Geographie darf sich nicht auf die Erfassung des Nahraums beschränken. Erdkunde umfaßt die ganze Erde. Es gibt kein Fach, welches die politisch wie wirtschaftlich notwendige Weltkenntnis vermitteln könnte.

\subsubsection{Zusätzliche Aufgaben}

\section{bei der Erfassung von Fernräumen}

1. Bei der Betrachtung eines Raumes müssen Wechselbeziehungen zwischen einzelnen Bereichen auch dann erfaßt werden, wenn man sich auf begrenzte Fragestellungen beschränkt. So läßt sich z. B. die agrarische Entwicklung nur erklären, wenn man neben der Betrachtung der Landwirtschaft auch die Politik der Industrialisierung besonders im ländlichen Raum, die Verflechtung von Stadt und Umland (Marktmöglichkeiten), die Migrationen und die Technisierung der Landwirtschaft einbezieht.

2. Es ist unerläßlich, die grundlegenden Wertvorstellungen des betreffenden Raumes wenigstens im Ansatz zu erkennen und zu berücksichtigen. Die Annahme eines "homo oeconomicus", eines stets oder zumindest vorwiegend rational Handelnden, hat sich als zu vordergründig erwiesen. Zwar sind wirtschaftliche Betrachtungsweisen global anzutreffen, doch werden sie durch regionale Wertsysteme vielfach abgewandelt.

Beispiele für regionale Wertsysteme sind

- das westeuropäische (Kennzeichen u. a. Marktwirtschaft, effiziente Technologie, Individualismus)

- das nordamerikanische («American Way of Life»)

- das ostasiatische (Konfuzianismus, Einordnung in eine Gemeinschaft, rationales Handeln)

- das islamische

Wertsysteme (Ideologien, Religionen) sind einerseits unerläßlich für ein Verständnis des (raumwirksamen) Handelns, andererseits bergen sie die große Gefahr, daß Prozesse unzulässig vereinfacht werden (z. B. «die» Schweizer, «die» Japaner, «der» Islam). Eine weitere Gefahr liegt darin, daß man von den eigenen Wertvorstellungen ausgeht und sie zum (alleinigen) Maßstab macht. Andererseits liegt auch im Gegenteil eine Gefahr: man akzeptiert jede Ideologie, läßt eigene Wertvorstellungen etwa über die Rechte des Individuums, der Frau im Zuge eines unverbindlichen Wertepluralismus zurücktreten. Gegenwärtig ist die Frage der Menschenrechte ein gutes Beispiel: Sollen sie global gültig sein, oder sind sie, da in Europa entstanden, auch nur hier und in den von Europäern besiedelten Räumen gültig?

Mit dem Einbeziehen der Ideen hinter den Prozessen gewinnt der Geographieunterricht eine neue Dimension. Er gewinnt auch eine erhöhte Bedeutung. 


\section{Literatur}

BIRKENHAUER, J. (1995): Zum Stand der Fachdidaktik der Geographie. In: Schulmagazin 5 bis 10, Heft 1/1995, S. 10-13.

BOHN, D. (1988): Allgemeine und/oder Regionale Geographie. In: Praxis Geographie, 18, Heft 7/8, 1988, S. 10-13.

HASSE, J. (1994): Die Suche nach dem «richtigen"Weg in der Erdkunde. Einige Anmerkungen zum philosophischen Postmodernismus. In: Geographie und ihre Didaktik, 22, Heft 3 1994 , S. 144-160.

HENNINGS, W. (1993): Strukturwandel und Bildungsreform. Überlegungen zu einer Revision der Curricula - auch in der Geographie. In: Geographie und ihre Didaktik, 21, Heft 3 1993, S. 122-141.

KLAFKI, W. (1991): Neue Studien zur Bildungstheorie und Didaktik. Weinheim/Basel.
KÖCK, H. (1993): Raumbezogene Schlüsselqualifikationen - der fachimmanente Beitrag des Geographieunterrichts zum Lebensalltag des Einzelnen und Funktionieren der Gesellschaft. In: Geographie und Schule, Heft 84, S. 14-22.

KROSS, E. (1992): Von der Inwertsetzung zur Bewahrung der Erde. Die curriculare Neuordnung des Geographieunterrichts. In Geographie heute, Heft 100, S. 57-62.

LEHRPLÄNE BAYERN. Bayerisches Staatsministerium für Unterricht, Kultus, Wissenschaft und Kunst. Lehrpläne Erdkunde: Hauptschule 1985 (Amtsblatt, Sondernummer 13); Realschule 1993 (Amtsblatt, Sondernummer 1); Gymnasium 1991 (Amtsblatt, Sondernummer 10).

NEWIG, J. (1995): Weltordnung und Kulturerdteile. In: Geographie heute, Heft 128, S. 44-45.

REINFRIED, S. (1994): Erfahrungen mit projektorientiertem Lernen im Geographieunterricht. In: Geographie und Schule, 16, Heft 92, S. 3-11.

SCHMITT-WULFFEN, W. (1994): "Schlüsselprobleme" als Grundlage künftigen Geographieunterrichts. In: Praxis Geographie, 24, Heft 3, 1994, S. 13-15.

STORKEBAUM, W. (1990): Länderkunde als curricularer Baustein. In: Praxis Geographie, 20, Heft 4, 1990, S. 8-12. 